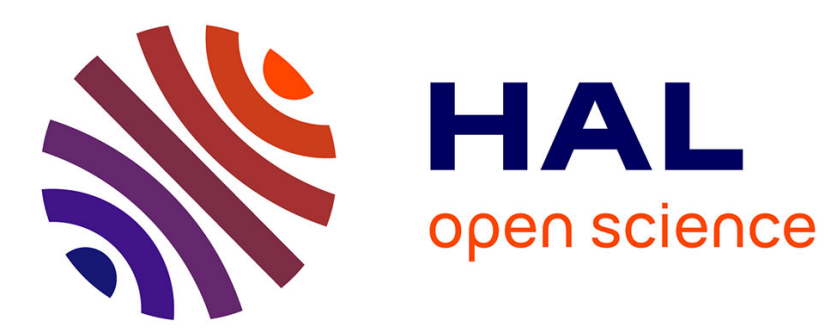

\title{
On the distribution of the summands of unequal partitions in residue classes
}

Cécile Dartyge, Andras Sarkozy, Mihaly Szalay

\section{To cite this version:}

Cécile Dartyge, Andras Sarkozy, Mihaly Szalay. On the distribution of the summands of unequal partitions in residue classes. Acta Mathematica Hungarica, 2006, 110 (4), pp.323-335. 10.1007/s10474006-0027-7 . hal-00091852

\section{HAL Id: hal-00091852 \\ https://hal.science/hal-00091852}

Submitted on 7 Sep 2006

HAL is a multi-disciplinary open access archive for the deposit and dissemination of scientific research documents, whether they are published or not. The documents may come from teaching and research institutions in France or abroad, or from public or private research centers.
L'archive ouverte pluridisciplinaire HAL, est destinée au dépôt et à la diffusion de documents scientifiques de niveau recherche, publiés ou non, émanant des établissements d'enseignement et de recherche français ou étrangers, des laboratoires publics ou privés. 


\title{
On the distribution of the summands of unequal partitions in residue classes
}

\author{
Cécile Dartyge, András Sárközy and Mihály Szalay *
}

\begin{abstract}
It is proved that the summands of almost all unequal partitions of $n$ are well-distributed modulo $d$ for $d=o\left(n^{1 / 2}\right)$.
\end{abstract}

\section{Introduction}

In [1], [2], [3] and [4] we studied some arithmetic properties of the parts of the partitions of an integer $n$. In [3] we proved that the summands of almost all partitions of $n$ are welldistributed modulo $d$ for $d$ up to $n^{1 / 2-\varepsilon}$. In this paper we will obtain a result of this type for partitions in unequal parts. Let $\mathcal{Q}(n)$ denote the set of the partitions of $n$ in unequal parts and $q(n)$ its cardinality (we set $q(0)=1$ ). Erdös and Lehner [5] proved that almost all of the $q(n)$ unequal partitions of $n$ contain

$$
(1+o(1)) \frac{2 \sqrt{3} \log 2}{\pi} \sqrt{n}
$$

parts. This is relatively small in comparison with the maximal summand in almost all unequal partitions of $n$. (By [5], both the number of parts and the maximal summand are equal to

$$
(1+o(1)) \frac{\sqrt{6}}{2 \pi} \sqrt{n} \log n
$$

in almost all "unrestricted" partitions of $n$.) Namely, it follows from Lemma 10 of [7] and the proof of Lemma 11 of [7] that the maximal summand is

$$
(1+o(1)) \frac{\sqrt{3}}{\pi} \sqrt{n} \log n
$$

in almost all unequal partitions of $n$. As a natural upper bound for the parts we shall use the double of the value of the above main term. We denote a general partition of $n$ with unequal parts by $\gamma=\left(\gamma_{1}, \ldots, \gamma_{s}\right)$ with $n=\gamma_{1}+\cdots+\gamma_{s}, \gamma_{1}>\ldots>\gamma_{s} \geqslant 1$. We will prove

2000 Mathematics Subject Classification 11P82,11P83

Key words and phrases: unequal partitions, residue classes.

* Research partially supported by the Hungarian National Foundation for Scientific Research,

Grant T043623 and by French-Hungarian EGIDE-OMKFHÁ exchange program Balaton F-2/03. 
Theorem 1.1. Let $\varepsilon>0,1 \leqslant r \leqslant d \leqslant \sqrt{n}, \Gamma \leqslant(2 \log n) \sqrt{3 n} / \pi$ and $w(n)$ be a nondecreasing function with $\lim _{n \rightarrow+\infty} w(n)=+\infty$. For all but $q(n) / w(n)$ partitions of $n$ with unequal parts we have

$$
\begin{aligned}
\sum_{\substack{\gamma_{j} \equiv r(\bmod d) \\
\gamma_{j} \geqslant \Gamma}} 1 & =(1+o(1)) \frac{2 \sqrt{3 n}}{\pi d} \log \left(1+\exp \left(-\frac{\pi\left(r+d\left(\Gamma^{\prime}-1\right)\right)}{2 \sqrt{3 n}}\right)\right)+O(1) \\
& +O\left(\sqrt{w(n)}\left(\frac{n^{3 / 8+\varepsilon}}{d}+\frac{n^{1 / 4}}{\sqrt{d}}\right)\right)
\end{aligned}
$$

where $\Gamma^{\prime}=\lceil(\Gamma-r) / d\rceil$.

The proof of this theorem is similar to the proofs of some results of [3] and [4]. We adopt a probabilistic approach. The only difference is that in some steps we will use the saddle point method. The parameter $\Gamma$ isn't crucial here. We have chosen to study the number of parts $\equiv r(\bmod d)$ and $\geqslant \Gamma$ only to have the same type of notations as in [3]. In the paper [3] about unrestricted partitions, this parameter was necessary because the number of small parts is preponderant. If we take $\Gamma=r$, we obtain

Corollary 1.2. Let $\varepsilon>0,1 \leqslant r \leqslant d \leqslant \sqrt{n}$ and $w(n)$ be a non-decreasing function with $\lim _{n \rightarrow+\infty} w(n)=+\infty$. For all but $q(n) / w(n)$ partitions of $n$ with unequal parts we have

$$
\begin{aligned}
\sum_{\gamma_{j} \equiv r(\bmod d)} 1 & =(1+o(1)) \frac{2 \sqrt{3 n}}{\pi d} \log \left(1+\exp \left(\frac{\pi(d-r)}{2 \sqrt{3 n}}\right)\right)+O(1) \\
& +O\left(\sqrt{w(n)}\left(\frac{n^{3 / 8+\varepsilon}}{d}+\frac{n^{1 / 4}}{\sqrt{d}}\right)\right)
\end{aligned}
$$

\section{A probabilistic approach}

For some integers $r, d, \Gamma$, with $1 \leqslant r \leqslant d, \Gamma \geqslant 1$ and a partition $\gamma \in \mathcal{Q}(n)$, let

$$
T(n, \gamma, \Gamma, r, d):=\sum_{\substack{\gamma_{j} \equiv r(\bmod d) \\ \gamma_{j} \geqslant \Gamma}} 1 .
$$

Let us consider the random field consisting of all possible choices of partitions of $n$ with unequal parts with equal probability. Let $\tau_{n}$ denote the random variable which assigns $T(n, \gamma, \Gamma, r, d)$ to a partition $\gamma \in \mathcal{Q}(n)$. We will need the estimates of the mean value $M\left(\tau_{n}\right)$ and the standard deviation $D\left(\tau_{n}\right)$ of $\tau_{n}$.

Let $q\left(n, a_{1}, \ldots, a_{k}\right)$ denote the number of unequal partitions of $n$ containing each $a_{j}$ as a summand. In the estimate of $M\left(\tau_{n}\right)$ and $D^{2}\left(\tau_{n}\right)$ we will use precise estimations of this quantity. Erdős and Szalay [9] proved the following lemma:

Lemma 2.1([9] Lemma 2 p. 96). Let $\varepsilon$ be fixed, $0<\varepsilon<10^{-2}$. For $1 \leqslant k \leqslant n^{1 / 6-\varepsilon}$ and $a_{1}+\cdots+a_{k} \leqslant n^{3 / 4-\varepsilon}$ we have

$$
q\left(n, a_{1}, \ldots, a_{k}\right)=(1+o(1)) \frac{q(n)}{\prod_{j=1}^{k}\left(1+\exp \left(\frac{\pi a_{j}}{2 \sqrt{3 n}}\right)\right)} .
$$

We will use this lemma to compute $M\left(\tau_{n}\right)$ but unfortunately the error term $o(1)$ in $(2 \cdot 2)$ is not sufficient to prove large enough cancellation in the estimate of $D^{2}\left(\tau_{n}\right)$. As it is said in [6] for the particular case $a_{i}=i(1 \leqslant i \leqslant k)$, this term can probably be replaced by $O\left(n^{-1 / 6+\varepsilon}\right)$ but this is still not sufficient for us. 


\section{The mean value $M\left(\tau_{n}\right)$}

In this paragraph we will prove

Lemma 3.1. For $1 \leqslant r \leqslant d \leqslant \sqrt{n}$ and $1 \leqslant \Gamma \leqslant 2 \frac{\sqrt{3 n}}{\pi} \log n$ we have

$$
M\left(\tau_{n}\right)=(1+o(1)) \frac{2 \sqrt{3 n}}{\pi d} \log \left(1+\exp \left(-\frac{\pi\left(r+d\left(\Gamma^{\prime}-1\right)\right)}{2 \sqrt{3 n}}\right)\right)+O(1)
$$

where $\Gamma^{\prime}=\left\lceil\frac{\Gamma-r}{d}\right\rceil$.

As in [3] and [4] we start with the formula

$$
T(n, \gamma, \Gamma, r, d)=\sum_{\substack{\Gamma^{\prime} \leqslant t \leqslant n^{\prime} \\ r+t d \in \gamma}} 1,
$$

where $n^{\prime}=\left\lfloor\frac{n-r}{d}\right\rfloor$. Let $s=\left\lfloor 10 \frac{\sqrt{3 n}}{\pi} \log n\right\rfloor+1, s^{\prime}=\left\lfloor\frac{s-r}{d}\right\rfloor$. We have

$$
\begin{aligned}
M\left(\tau_{n}\right) & =\frac{1}{q(n)} \sum_{\gamma \in \mathcal{Q}(n)} T(n, \gamma, \Gamma, r, d)=\frac{1}{q(n)} \sum_{t=\Gamma^{\prime}}^{n^{\prime}} \sum_{\substack{\gamma \in \mathcal{Q}(n) \\
r+t d \in \gamma}} 1 \\
& =\frac{1}{q(n)} \sum_{t=\Gamma^{\prime}}^{n^{\prime}} q(n, r+t d) \\
& =\frac{1}{q(n)} \sum_{t=\Gamma^{\prime}}^{s^{\prime}} q(n, r+t d)+\frac{1}{q(n)} \sum_{t=s^{\prime}+1}^{n^{\prime}} q(n, r+t d) \\
& =S_{1}+S_{2},
\end{aligned}
$$

say. First we give an upper bound for $S_{2}$. The partitions counted in this term have at least one big part. Since

$$
q(n, r+t d) \leqslant q(n-r-t d)
$$

we have

$$
S_{2} \leqslant \frac{1}{q(n)} \sum_{t=s^{\prime}+1}^{n^{\prime}} q(n-r-t d) .
$$

Hardy and Ramanujan [10] proved the formula

$$
q(n) \sim \frac{1}{4\left(3 n^{3}\right)^{1 / 4}} \exp \left(\pi \sqrt{\frac{n}{3}}\right) .
$$

The function $q(n)$ is non-decreasing on $n$. Thus for $t>s^{\prime}$ we have

$$
q(n-r-t d) \leqslant q(n-s) \ll q(n) \exp \left(\frac{\pi}{\sqrt{3}}(\sqrt{n-s}-\sqrt{n})\right) \ll q(n) \exp \left(-\frac{\pi s}{2 \sqrt{3 n}}\right) .
$$

By $(3 \cdot 2)$ and $(3 \cdot 4)$ we have

$$
S_{2} \ll n \exp (-5 \log n) \ll n^{-4}
$$


The term $S_{1}$ is the main contribution to $M\left(\tau_{n}\right)$. By Lemma 2.1 we have:

$$
S_{1}=(1+o(1)) \sum_{t=\Gamma^{\prime}}^{s^{\prime}} \frac{1}{1+\exp \left(\frac{\pi(r+t d)}{2 \sqrt{3 n}}\right)} .
$$

We evaluate the above sum with a standard lemma which compares a series with an integral (see for example [11], p. 4).

Lemma 3.2. Let $f:[a, b] \rightarrow \mathbb{R}$ with $a, b \in \mathbb{Z}$ a monotonic function. There exists $\vartheta=\vartheta(a, b), 0 \leqslant \vartheta \leqslant 1$ such that

$$
\sum_{a<n \leqslant b} f(n)=\int_{a}^{b} f(t) d t+\vartheta(f(b)-f(a)) .
$$

We apply this with $a=\Gamma^{\prime}-1, b=s^{\prime}$ and

$$
f(t)=\left(1+\exp \left(\frac{\pi(r+t d)}{2 \sqrt{3 n}}\right)\right)^{-1} .
$$

We observe that $f(t)<1$ for all $t$ 's. Thus we have

$$
\begin{aligned}
S_{1} & =(1+o(1)) \int_{\Gamma^{\prime}-1}^{s^{\prime}} \frac{d t}{1+\exp \left(\frac{\pi(r+t d)}{2 \sqrt{3 n}}\right)}+O(1) \\
& =(1+o(1)) \frac{2 \sqrt{3 n}}{\pi d}\left(\log \left(1-f\left(s^{\prime}\right)\right)-\log \left(1-f\left(\Gamma^{\prime}-1\right)\right)\right)+O(1)
\end{aligned}
$$

and since $f\left(s^{\prime}\right)=o\left(f\left(\Gamma^{\prime}-1\right)\right)$,

$$
S_{1}=(1+o(1)) \frac{2 \sqrt{3 n}}{\pi d} \log \left(1+\exp \left(-\frac{\pi\left(r+d\left(\Gamma^{\prime}-1\right)\right)}{2 \sqrt{3 n}}\right)\right)+O(1) .
$$

We get Lemma 3.1 from $(3 \cdot 7)$ and $(3 \cdot 5)$.

\section{The standard deviation}

In this paragraph we will prove

Lemma 4.1. Let $\varepsilon>0$. For $1 \leqslant r \leqslant d \leqslant \sqrt{n}$ and $1 \leqslant \Gamma \leqslant 2 \frac{\sqrt{3 n}}{\pi} \log n$ we have

$$
D^{2}\left(\tau_{n}\right) \ll \frac{\sqrt{n}}{d}+\frac{n^{\frac{3}{4}+\varepsilon}}{d^{2}} .
$$

We start with the well-known formula $D^{2}\left(\tau_{n}\right)=M\left(\tau_{n}^{2}\right)-M\left(\tau_{n}\right)^{2}$. To compute $M\left(\tau_{n}\right)^{2}$ we develop the square of $(3 \cdot 1) ;(3 \cdot 6)$ yields $S_{1}=O(n)$ and we use $(3 \cdot 5)$ :

$$
\begin{aligned}
M\left(\tau_{n}\right)^{2} & =\left(S_{1}+S_{2}\right)^{2} \\
& =\frac{1}{q(n)^{2}}\left(\sum_{\Gamma^{\prime} \leqslant t \leqslant s^{\prime}} q(n, r+t d)\right)^{2}+O\left(n^{-3}\right) \\
& =\frac{1}{q(n)^{2}} \sum_{\substack{\Gamma^{\prime} \leqslant t_{1}, t_{2} \leqslant s^{\prime} \\
t_{1} \neq t_{2}}} q\left(n, r+t_{1} d\right) q\left(n, r+t_{2} d\right) \\
& +\frac{1}{q(n)^{2}} \sum_{\Gamma^{\prime} \leqslant t \leqslant s^{\prime}} q(n, r+t d)^{2}+O\left(n^{-3}\right) .
\end{aligned}
$$


Remark 1. The last sum in $(4 \cdot 1)$ satisfies

$$
S_{1}^{\prime}:=\frac{1}{q(n)^{2}} \sum_{\Gamma^{\prime} \leqslant t \leqslant s^{\prime}} q(n, r+t d)^{2} \leqslant S_{1}=O\left(M\left(\tau_{n}\right)\right) .
$$

Next we compute $M\left(\tau_{n}^{2}\right)$ in the same way :

$$
\begin{aligned}
M\left(\tau_{n}^{2}\right) & =\frac{1}{q(n)} \sum_{\gamma \in \mathcal{Q}(n)} T^{2}(n, \gamma, \Gamma, r, d)=\frac{1}{q(n)} \sum_{\substack{\gamma \in \mathcal{Q}(n) \\
t_{1}, t_{2} \geqslant \Gamma^{\prime} \\
r+t_{1} d \in \gamma \\
r+t_{2} d \in \gamma}} 1 \\
& =\frac{1}{q(n)} \sum_{\substack{t_{1}, t_{2} \geqslant \Gamma^{\prime} \\
t_{1} \neq t_{2}}} q\left(n, r+t_{1} d, r+t_{2} d\right)+\frac{1}{q(n)} \sum_{\substack{\Gamma^{\prime} \leqslant t \leqslant n^{\prime} \\
\Gamma^{\prime}}} q(n, r+t d) .
\end{aligned}
$$

With the same arguments as the one used for $S_{2}$, we see that the contribution of the terms $\max \left(t_{1}, t_{2}\right) \geqslant s^{\prime}$ is small enough :

$$
M\left(\tau_{n}^{2}\right)=\frac{1}{q(n)} \sum_{\substack{\Gamma^{\prime} \leqslant t_{1}, t_{2} \leqslant s^{\prime} \\ t_{1} \neq t_{2}}} q\left(n, r+t_{1} d, r+t_{2} d\right)+M\left(\tau_{n}\right)+O\left(n^{-3}\right) .
$$

We will see that the leading terms in $(4 \cdot 1)$ and $(4 \cdot 3)$ cancel out. Unfortunately Lemma 2.1 is not precise enough to guarantee that $D^{2}\left(\tau_{n}\right)=O\left(M\left(\tau_{n}\right)\right)$. We should have $O\left(n^{-\alpha}\right)$ with some $\alpha>1 / 2$ instead of $o(1)$ in $(2 \cdot 2)$ to be able to state Lemma 4.1. Erdős, Nicolas and Szalay [6] proved a version of Lemma 2.1 in the case $a_{j}=j$ for $1 \leqslant j \leqslant k$. In this paper (page 21 of [6]) there is a very precise asymptotic formula of $q(n)$. With this formula we could compute $q(n-t) / q(n)$ (like the estimate of $p(n-t) / p(n)$ we obtained in Lemma 3 in [3]) and after use the inclusion-exclusion principle to obtain an estimation of $q\left(n, r+t_{1} d, r+t_{2} d\right)$ with the desired precision. The price to pay for applying this method is that the computations are long.

In this paper we will use the saddle point method to obtain an upper bound in the critical range $\Gamma^{\prime} \leqslant t_{1}, t_{2} \leqslant s^{\prime}, t_{1} \neq t_{2}$ for the function

$$
Q\left(a_{1}, a_{2}\right):=\frac{q\left(n, a_{1}, a_{2}\right)}{q(n)}-\frac{q\left(n, a_{1}\right) q\left(n, a_{2}\right)}{q(n)^{2}}
$$

where $a_{1}=r+t_{1} d$ and $a_{2}=r+t_{2} d$. There are two advantages to apply here he saddle point method. The first one is that in different steps we can use the precise estimates of Erdős, Nicolas and Szalay [8], [9], [6]. The second one is that the compensations between the main terms of $q\left(n, a_{1}, a_{2}\right) / q(n)$ and $q\left(n, a_{1}\right) q\left(n, a_{2}\right) / q(n)^{2}$ are more easy to see if we use integral representations.

Let $h$ denote the function defined for $x=\Re z>0$ by

$$
h(z)=\prod_{\nu=1}^{\infty}(1+\exp (-\nu z)) .
$$

We also define

$$
F\left(z, a_{1}, \ldots, a_{k}\right):=h(z) \prod_{j=1}^{k}\left(1+\exp \left(-a_{j} z\right)\right)^{-1} \exp \left(\left(n-\sum_{j=1}^{k} a_{j}\right) z\right) .
$$


We have for any $x_{0}>0$

$$
q\left(n, a_{1}, \ldots, a_{k}\right)=\frac{1}{2 \pi} \int_{-\pi}^{\pi} F\left(x_{0}+i y, a_{1}, \ldots, a_{k}\right) d y \leqslant q(n) .
$$

We adopt some other notations of the papers [6], [8] and [9] :

$$
x_{0}=\frac{\pi}{2 \sqrt{3 n}}, \quad y_{1}=n^{-3 / 4+\varepsilon / 3} \quad \text { and } \quad y_{2}=c_{0} x_{0}
$$

with $c_{0}$ sufficiently large and $\left.\varepsilon \in\right] 0,10^{-2}$ [ fixed. For small $y$, one might like to substitute $1+\exp \left(-a\left(x_{0}+i y\right)\right)$ by $1+\exp \left(-a x_{0}\right)$ as in [9], p. 102-103. Lemma 4.2 and Lemma 4.3 require simple ratio estimates collected in the following remark.

Remark 2. Let $-\pi \leqslant y \leqslant \pi$.

(i) For $a \geqslant 1$ and $|y| \leqslant x_{0} / 2$, we have

$$
\left|\frac{1+\exp \left(-a x_{0}\right)}{1+\exp \left(-a\left(x_{0}+i y\right)\right)}\right|=\frac{1}{\left|1-\frac{1-\exp (-i a y)}{1+\exp \left(a x_{0}\right)}\right|} \leqslant \frac{1}{1-\frac{|1-\exp (-i a y)|}{1+\exp \left(a x_{0}\right)}} \leqslant \frac{1}{1-\frac{a|y|}{a x_{0}}} \leqslant 1+2 \frac{|y|}{x_{0}} \text {. }
$$

(ii) For $a \geqslant 1$, we have

$$
\left|\frac{1+\exp \left(-a x_{0}\right)}{1+\exp \left(-a\left(x_{0}+i y\right)\right)}\right| \leqslant \frac{1}{1-\frac{|1-\exp (-i a y)|}{1+\exp \left(a x_{0}\right)}} \leqslant \frac{1}{1-\frac{2}{1+\exp \left(x_{0}\right)}}<\frac{4}{x_{0}} .
$$

(iii) For $1 \leqslant a \leqslant 1 /\left(2 y_{1}\right)$ and $|y| \leqslant y_{1}$, we have

$$
\left|\frac{1+\exp \left(a x_{0}\right)}{1+\exp \left(a\left(x_{0}+i y\right)\right)}\right|=\frac{1}{\left|1-\frac{1-\exp (i a y)}{1+\exp \left(-a x_{0}\right)}\right|} \leqslant \frac{1}{1-\frac{a|y|}{1+\exp \left(-a x_{0}\right)}} \leqslant \frac{1}{1-a|y|} \leqslant 2 .
$$

It can be shown by combining proofs in the different papers of Erdős, Nicolas and Szalay [8], [9] and [6] that

Lemma 4.2. For any fixed $k$, there exists $c>0$ such that for $\max \left(a_{1}, \ldots, a_{k}\right)<s$ we have

$$
q\left(n, a_{1}, \ldots, a_{k}\right)=\frac{1}{2 \pi} \int_{|y| \leqslant y_{1}} F\left(x_{0}+i y, a_{1}, \ldots, a_{k}\right) d y+O\left(q(n) \exp \left(-c n^{2 \varepsilon / 3}\right)\right) .
$$

We give only the outlines of the proof but we indicate the references for the details. We cut the integral $(4 \cdot 5)$ in three parts :

$$
\begin{aligned}
q\left(n, a_{1}, \ldots, a_{k}\right) & =\frac{1}{2 \pi} \int_{|y| \leqslant y_{1}} F\left(x_{0}+i y, a_{1}, \ldots, a_{k}\right) d y \\
& +\frac{1}{2 \pi} \int_{y_{1}<|y| \leqslant y_{2}} F\left(x_{0}+i y, a_{1}, \ldots, a_{k}\right) d y \\
& +\frac{1}{2 \pi} \int_{y_{2}<|y| \leqslant \pi} F\left(x_{0}+i y, a_{1}, \ldots, a_{k}\right) d y .
\end{aligned}
$$

We will prove that the two last integrals are small enough. Erdős and Szalay used the formula (cf. (4.3) and (4.4) of [8])

$$
h(z)=\exp \left(\frac{\pi^{2}}{12 z}-\frac{\log 2}{2}+o(1)\right) \quad \text { for } z \rightarrow 0 \text { in }|\arg z| \leqslant \kappa<\pi / 2 .
$$


For $y_{1} \leqslant|y| \leqslant y_{2},(4 \cdot 6)$ implies that

$$
\begin{aligned}
\left|h\left(x_{0}+i y\right)\right| & \ll \exp \left(\frac{\pi^{2}}{12} \Re \frac{1}{x_{0}+i y}\right)=\exp \left(\frac{\pi^{2} x_{0}}{12\left(x_{0}^{2}+y^{2}\right)}\right) \\
& \leqslant \exp \left(\frac{\pi^{2} x_{0}}{12\left(x_{0}^{2}+y_{1}^{2}\right)}\right)=\exp \left(\frac{\pi^{2}}{12 x_{0}}\left(1-\frac{y_{1}^{2}}{x_{0}^{2}}+O\left(\frac{y_{1}^{4}}{x_{0}^{4}}\right)\right)\right) \\
& \ll \exp \left(\frac{\pi^{2}}{12 x_{0}}-\frac{2 \sqrt{3}}{\pi} n^{2 \varepsilon / 3}\right) .
\end{aligned}
$$

Then (4.7) and Remark 2 (ii) yield that

$$
\left|F\left(x_{0}+i y, a_{1}, \ldots, a_{k}\right)\right| \ll\left(\frac{4}{x_{0}}\right)^{k} \exp \left(n x_{0}+\frac{\pi^{2}}{12 x_{0}}-\frac{2 \sqrt{3}}{\pi} n^{2 \varepsilon / 3}\right)
$$

and we obtain

$$
\frac{1}{2 \pi} \int_{y_{1}<|y| \leqslant y_{2}} F\left(x_{0}+i y, a_{1}, \ldots, a_{k}\right) d y \ll q(n) \exp \left(-c n^{2 \varepsilon / 3}\right),
$$

for $c>0$ small enough.

For $y_{2}<|y| \leqslant \pi$, Erdős and Szalay [8], p. 439 showed the upper bound

$$
\left|h\left(x_{0}+i y\right)\right| \leqslant \exp \left(\frac{1}{x_{0}}\left(\frac{\pi^{2}}{6}-1+\frac{\pi}{2 c_{0}}\right)\right) .
$$

Using this (with $c_{0}=6 \pi$, say) and Remark 2 (ii) we obtain (cf. p. 440 of [8]):

$$
\frac{1}{2 \pi} \int_{y_{2}<|y| \leqslant \pi} F\left(x_{0}+i y, a_{1}, \ldots, a_{k}\right) d y \leqslant q(n) \exp \left(-c^{\prime} n^{1 / 2}\right)
$$

for some $c^{\prime}>0$ small enough. These two upper bounds (4.8), (4.9) are sufficient to prove Lemma 4.2.

The error term $o(1)$ in Lemma 2.1 is a consequence of the contribution of the range $|y| \leqslant y_{1}$. Now we return to the function $Q\left(a_{1}, a_{2}\right)$.

Lemma 4.3. There exists $c>0$ small enough such that for $0 \leqslant a_{1}, a_{2} \leqslant s, a_{1} \neq a_{2}$, we have:

$$
Q\left(a_{1}, a_{2}\right) \ll \frac{y_{1} a_{2} n^{2 \varepsilon}}{\left(1+\exp \left(x_{0} a_{2}\right)\right)\left(1+\exp \left(x_{0} a_{1}\right)\right)}+O\left(\exp \left(-c n^{2 \varepsilon / 3}\right)\right) .
$$

By the Cauchy formula we have :

$$
\begin{aligned}
Q\left(a_{1}, a_{2}\right)= & \frac{q\left(n, a_{1}, a_{2}\right) q(n)}{q(n)^{2}}-\frac{q\left(n, a_{1}\right) q\left(n, a_{2}\right)}{q(n)^{2}} \\
= & \frac{1}{4 \pi^{2} q(n)^{2}} \int_{-\pi}^{\pi} F\left(x_{0}+i y, a_{1}, a_{2}\right) d y \\
& \times \int_{-\pi}^{\pi} h\left(x_{0}+i y^{\prime}\right) \exp \left(n\left(x_{0}+i y^{\prime}\right)\right) d y^{\prime} \\
- & \frac{1}{4 \pi^{2} q(n)^{2}} \int_{-\pi}^{\pi} F\left(x_{0}+i y, a_{1}\right) d y \int_{-\pi}^{\pi} F\left(x_{0}+i y^{\prime}, a_{2}\right) d y^{\prime} .
\end{aligned}
$$


In the four above integrals, by (4.5) and Lemma 4.2 the contribution of the range $\max \left(|y|,\left|y^{\prime}\right|\right)>y_{1}$ is $O\left(\exp \left(-c n^{2 \varepsilon / 3}\right)\right.$ for some $c>0$. Let $H\left(a_{1}, y, y^{\prime}\right)$ be the function defined by

$$
\begin{aligned}
H\left(a_{1}, y, y^{\prime}\right) & =\frac{h\left(x_{0}+i y\right) h\left(x_{0}+i y^{\prime}\right)}{4 \pi^{2} q(n)^{2}}\left(1+\exp \left(-a_{1}\left(x_{0}+i y\right)\right)\right)^{-1} \\
& \times \exp \left(\left(n-a_{1}\right)\left(x_{0}+i y\right)\right) \exp \left(n\left(x_{0}+i y^{\prime}\right)\right)
\end{aligned}
$$

and $\varphi$ the function

$$
\varphi\left(a_{2}, y\right)=\frac{\exp \left(-\left(x_{0}+i y\right) a_{2}\right)}{1+\exp \left(-a_{2}\left(x_{0}+i y\right)\right)}=\frac{1}{1+\exp \left(a_{2}\left(x_{0}+i y\right)\right)} .
$$

With this notation and by Lemma 4.2 we have

$$
Q\left(a_{1}, a_{2}\right)=\iint_{\max \left(|y|,\left|y^{\prime}\right|\right) \leqslant y_{1}} H\left(a_{1}, y, y^{\prime}\right)\left(\varphi\left(a_{2}, y\right)-\varphi\left(a_{2}, y^{\prime}\right)\right) d y d y^{\prime}+O\left(\exp \left(-c n^{2 \varepsilon / 3}\right)\right) .
$$

By standard computations it follows for $\max \left(|y|,\left|y^{\prime}\right|\right) \leqslant y_{1}$ :

$$
\begin{aligned}
\varphi\left(a_{2}, y\right)-\varphi\left(a_{2}, y^{\prime}\right) & =\frac{\mathrm{e}^{x_{0} a_{2}}\left(\mathrm{e}^{i y^{\prime} a_{2}}-\mathrm{e}^{i y a_{2}}\right)}{\left(1+\exp \left(\left(x_{0}+i y\right) a_{2}\right)\right)\left(1+\exp \left(\left(x_{0}+i y^{\prime}\right) a_{2}\right)\right)} \\
& =\frac{\mathrm{e}^{x_{0} a_{2}}\left(i\left(y^{\prime}-y\right) a_{2}+O\left(y_{1}^{2} a_{2}^{2}\right)\right)}{\left(1+\exp \left(\left(x_{0}+i y\right) a_{2}\right)\right)\left(1+\exp \left(\left(x_{0}+i y^{\prime}\right) a_{2}\right)\right)} .
\end{aligned}
$$

By Remark 2 (iii), we have for $|y| \leqslant y_{1}$ :

$$
\left|1+\exp \left(\left(x_{0}+i y\right) a_{2}\right)\right|^{-1} \ll\left(1+\exp \left(x_{0} a_{2}\right)\right)^{-1} .
$$

Since $y_{1} s=o(1)$, this gives for $\max \left(|y|,\left|y^{\prime}\right|\right) \leqslant y_{1}$ :

$$
\left|\varphi\left(a_{2}, y\right)-\varphi\left(a_{2}, y^{\prime}\right)\right| \ll \frac{y_{1} a_{2}}{1+\exp \left(x_{0} a_{2}\right)}
$$

By Remark 2 (i), (4·6) and (3·3), for $\max \left(|y|,\left|y^{\prime}\right|\right) \leqslant y_{1}$ we have :

$$
\begin{aligned}
\left|H\left(a_{1}, y, y^{\prime}\right)\right| & \ll \frac{\exp \left(\frac{\pi^{2}}{6 x_{0}}+2 n x_{0}\right)}{q(n)^{2}\left(1+\exp \left(x_{0} a_{1}\right)\right)} \\
& \ll \frac{n^{3 / 2}}{1+\exp \left(x_{0} a_{1}\right)} .
\end{aligned}
$$

Inserting (4.13) and $(4 \cdot 14)$ in $(4 \cdot 12)$ we find

$$
Q\left(a_{1}, a_{2}\right) \ll \frac{y_{1} a_{2} n^{2 \varepsilon}}{\left(1+\exp \left(x_{0} a_{2}\right)\right)\left(1+\exp \left(x_{0} a_{1}\right)\right)}+O\left(\exp \left(-c n^{2 \varepsilon / 3}\right)\right) .
$$

The proof of Lemma 4.3 is now complete. 
By this Lemma, (3·7), (4.1), Remark 1, (4·3) and Lemma 3.1 we have

$$
\begin{aligned}
D^{2}\left(\tau_{n}\right) & \ll \sum_{\substack{\Gamma^{\prime} \leqslant t_{1}, t_{2} \leqslant s^{\prime} \\
t_{1} \neq t_{2}}} Q\left(r+t_{1} d, r+t_{2} d\right)+O\left(M\left(\tau_{n}\right)\right) \\
& \ll \sum_{\substack{\Gamma^{\prime} \leqslant t_{1}, t_{2} \leqslant s^{\prime} \\
t_{1} \neq t_{2}}} \frac{y_{1}\left(r+t_{2} d\right) n^{2 \varepsilon}}{\left(1+\exp \left(x_{0}\left(r+t_{2} d\right)\right)\right)\left(1+\exp \left(x_{0}\left(r+t_{1} d\right)\right)\right)}+O\left(\frac{\sqrt{n}}{d}\right) \\
& \ll \sum_{\substack{t_{1}, t_{2} \leqslant s^{\prime} \\
n_{1}}} y_{1}\left(r+t_{2} d\right) n^{2 \varepsilon}+\frac{\sqrt{n}}{d} \\
& \ll \frac{n^{3 / 4+4 \varepsilon}}{d^{2}}+\frac{\sqrt{n}}{d} .
\end{aligned}
$$

This ends the proof of Lemma 4.1.

\section{Completion of the proof of Theorem 1.1}

Let $w$ be a non-decreasing function with $\lim _{n \rightarrow \infty} w(n)=\infty$. Applying Chebyshev's inequality as we did in [3] and [4], we obtain that for all but $q(n) / w(n)$ partitions of $n$ with unequal parts we have

$$
\sum_{\substack{\gamma_{j} \equiv r(\bmod d) \\ \gamma_{j} \geqslant \Gamma}} 1=M\left(\tau_{n}\right)+O\left(\sqrt{D^{2}\left(\tau_{n}\right) w(n)}\right) .
$$

By Lemma 3.1 and Lemma 4.1 we have

$$
\begin{aligned}
\sum_{\substack{\gamma_{j} \equiv r(\bmod d) \\
\gamma_{j} \geqslant \Gamma}} 1 & =(1+o(1)) \frac{2 \sqrt{3 n}}{\pi d} \log \left(1+\exp \left(-\frac{\pi\left(r+d\left(\Gamma^{\prime}-1\right)\right)}{2 \sqrt{3 n}}\right)\right)+O(1) \\
& +O\left(\sqrt{w(n)}\left(\frac{n^{3 / 8+\varepsilon}}{d}+\frac{n^{1 / 4}}{\sqrt{d}}\right)\right)
\end{aligned}
$$

which completes the proof of Theorem 1.1.

\section{Further problems}

In this paper so far we have studied only the number of the parts belonging to a given residue class but not their sum. In [1] and [3] in case of unrestricted partitions we also showed that the sum of the parts belonging to a residue class modulo $d$ is well distributed modulo $d$, for almost all partitions of $n, i$. e., it is $(1+o(1)) n / d$ if $d$ is "not very large". It could be shown by using the same probabilistic method as in the proof of Theorem 1.1 above that this is so in case of unequal partitions as well.

We expect that in case of unrestricted partitions the distribution of the parts in residue classes is "less uniform" than in case of unequal partitions; this slight irregularity is due to the fact that in case of unrestricted partitions the "small" parts occur more frequently. We will say that the residue class $a(\bmod d)$ dominates the residue class $b(\bmod d)$ in a partition if there are more parts $\equiv a(\bmod d)$ than parts $\equiv b(\bmod d)$, and that the residue class $a(\bmod d)$ is a champion relative to a given partition if the residue class $a(\bmod$ d) contains at least as many summands of the partition as any other residue class. We conjecture that if $d$ is fixed, then in case of unrestricted partitions there is a $c=c(d)>0$ so that if $1 \leqslant a<b \leqslant d$ and $n>n_{0}(d)$, then the residue class $a(\bmod d)$ dominates the residue class $b(\bmod d)$ in more than $(1 / 2+c) p(n)$ partitions ; on the other hand, 
in case of unequal partitions, if $1 \leqslant a<b \leqslant d$ and $n \rightarrow \infty$ then the residue class $a$ $(\bmod d)$ dominates the residue class $b(\bmod d)$ in $(1 / 2+o(1)) q(n)$ partitions. Moreover, we conjecture that in case of unrestricted partitions there is a $c^{\prime}=c^{\prime}(d)>0$ so that the residue class 1 is a champion in more than $\left(1 / d+c^{\prime}\right) p(n)$ partitions, while in case of unequal partitions every residue class mod $d$ is a champion in $(1 / d+o(1)) q(n)$ partitions. In [2], we obtained results of this type but only with $c=c^{\prime}=0$. We will return to these questions in a subsequent paper.

In [2] and [4] in case of unrestricted partitions we utilized our well distribution results by studying certain arithmetic properties of the parts of random partitions : we studied the rate of the square-free parts to all parts, the maximum of the $\omega$ and $\Omega$ functions over the parts, and we also proved a Hardy-Ramanujan type theorem about the values of $\omega$ assumed over the parts. In the unequal case we could prove similar results in similar manner; since both the results and the methods are nearly the same, thus we do not present the details here.

On the other hand, there are arithmetic properties whose behaviour is significantly different in the unrestricted, resp. unequal case; we expect that this is so, e. g., in case of the rate of the prime parts to all parts of the partition. We will analyze this problem in a subsequent paper.

\section{References}

[1] C. Dartyge and A. Sárközy, Arithmetic properties of summands of partitions, to appear in Ramanujan Journal.

[2] C. Dartyge and A. Sárközy, Arithmetic properties of summands of partitions, II, to appear in Ramanujan Journal.

[3] C. Dartyge, A. Sárközy and M. Szalay, On the distribution of the summands of partitions in residue classes, to appear in Acta Math. Hungar.

[4] C. Dartyge, A. Sárközy and M. Szalay, On the number of prime factors of summands of partitions, preprint.

[5] P. Erdős and J. Lehner, The distribution of the number of summands in the partitions of a positive integer, Duke Math. Journal, 8 (1941), 335-345.

[6] P. Erdős, J.-L. Nicolas and M. Szalay, Partitions into parts which are unequal and large, Number theory (Ulm, 1987), 19-30, Lecture Notes in Math., 1380, Springer, New York, 1989.

[7] P. Erdős and M. Szalay, On some problems of J. Dénes and P. Turán, in: Studies in Pure Mathematics (to the Memory of Paul Turán), 187-212, Akadémiai Kiadó, Budapest, 1983.

[8] P. Erdős and M. Szalay, On the statistical theory of partitions, In : Coll. Math. Soc. János Bolyai 34. Topics in Classical Number Theory (Budapest 1981), 397-450, North-Holland/Elsevier, 1984.

[9] P. Erdős and M. Szalay, On some problems of the statistical theory of partitions, In: Number theory, Vol. I (Budapest, 1987), 93-110, Colloq. Math. Soc. János Bolyai, 51, North-Holland, Amsterdam, 1990.

[10] G. H. Hardy and S. Ramanujan, Asymptotic formulae in combinatory analysis, Proc. London Math. Soc., 17 (1918), 75-115.

[11] G. Tenenbaum, Introduction à la théorie analytique et probabiliste des nombres, 2 ème édition, Cours spécialisés, $\mathrm{n}^{\circ} 1$, Société mathématique de France (1995).

Cécile Dartyge

Institut Élie Cartan

Université Henri Poincaré-Nancy 1

BP 239

54506 Vandouvre Cedex

France

dartyge@iecn.u-nancy.fr
András Sárközy and Mihály Szalay Department of Algebra and Number Theory

Eötvös Loránd University

H-1117 Budapest

Pázmány Péter sétány 1/C

Hungary

sarkozy@cs.elte.hu

mszalay@cs.elte.hu 